\title{
Vibration and acoustic radiation of bogie area under random excitation in high-speed trains
}

\author{
Dongzhen Wang ${ }^{1,2}$ (D) $\cdot$ Jianmin $\mathrm{Ge}^{1}$
}

Received: 5 November 2018/Revised: 13 February 2019/Accepted: 16 February 2019/Published online: 19 March 2019

(C) The Author(s) 2019

\begin{abstract}
Based on the experiments on a platform with real vehicle structure and finite element simulation, the vibration and interior acoustic radiation under random excitations of high-speed trains' bogie area were studied. Firstly, combined with line tests, a vehicle body with a length of $7 \mathrm{~m}$ was used as the research object. By comparing the results of experiment and simulation, the accuracy of the finite element model was verified. Secondly, the power spectral density curves at typical measuring points in bogie area were obtained by processing and calculating the line test data, which was measured when the vehicle ran at high speeds, and the standard vibration spectrum of the bogie area was obtained by the extreme envelope method. Furthermore, the random vibration test and simulation prediction analysis of the real vehicle structure were carried out to further verify the accuracy of the noise and vibration prediction model. Finally, according to the vibration and acoustic radiation theory, the indirect boundary element method was adopted to predict the acoustic response of the real vehicle. The analysis shows that the simulated power spectral density curves of acceleration and sound pressure level are highly consistent with the experimental ones, and the error between the simulated prediction and the experimental result is within the allowable range of $3 \mathrm{~dB}$.
\end{abstract}

Dongzhen Wang

wangdongzhen@cqsf.com

1 School of Physical Sciences and Engineering, Tongji University, Shanghai 200092, China

2 CRRC Qingdao Sifang Co., Ltd, Qingdao 266111, China
Keywords High-speed trains - Standard vibration spectrum $\cdot$ Indirect boundary element method $\cdot$ Random excitation $\cdot$ Acoustic radiation

\section{Introduction}

With the rapid development of high-speed trains, the vibration and noise problem has become increasingly prominent. The vehicle body needs to have sufficient static stiffness to maintain the bearing capacity and reasonable dynamic characteristics to keep the vibration and noise under control. Because the excitation exerted on the car body has obvious randomness, the generated radiation noise is also random. As the wheel-rail excitation and aerodynamic excitation in the bogie area are the main sources of vehicle vibration, the study of excitation characteristics and acoustic radiation response characteristics of the vehicle in the bogie area is of great significance for controlling the noise of high-speed trains.

Liu et al. [1] proposed a statistical boundary method combining boundary element and statistical methods. Allen et al. [2,3] combined finite element method and boundary element method to solve the random sound field and verified the feasibility of their method through experiment and simulation analyses. Li et al. [4] applied the finite element method to calculate the random sound field response of the thin plate excited by the random load, which showed that the acoustic response characteristics of the system depend on the acoustic frequency response function and the power spectral density (PSD) function. Liu et al. [5, 6] studied the acoustic radiation sensitivity and optimization design under random excitation using finite element method, boundary element method and virtual excitation method. Zhao et al. [7] applied a new algorithm to calculate the vibration and 
sound radiation of the structure under random excitation using the virtual excitation method. Mehran et al. [8] analysed the random vibration and sound radiation of the rail carriage with ABAQUS software.

To date, many studies have been carried out on the problems of vibration and sound radiation of car body under certain excitations [9-11], but there is less research work on vibration and acoustic radiation under random excitation, and most of the work focuses on simulation. Combing the test with simulation method for real vehicle structure, we applied the finite element and indirect boundary element methods to study the vibration and noise of the vehicle body for a high-speed train under the random excitation from the bogie. A set of simple, practical, fast and accurate noise prediction methods for the high-speed train has been explored, which can provide more accurate acoustic characteristics evaluation at the beginning of design stage.

\section{Finite element modelling and verification for vehicle body}

\subsection{Selection of research object}

The main noise sources of high-speed trains include wheel-rail noise, aerodynamic noise and equipment noise. In contrast to the central part of carriage, the noise generated from the both sides of the carriage is more difficult to control, due to the direct influence of the wheel-rail noise and aerodynamic noise coming from the bogie area. Therefore, the intensity of the noise and vibration produced from the bogie area determines the reduction effect of the vibration and noise from the both sides of saloon.

To this end, the longitudinal distribution characteristics of interior noise were studied for the train at the speed of $350 \mathrm{~km} / \mathrm{h}$. Some measuring points were arranged along longitudinal direction of the vehicle, which are at a height of $1.2 \mathrm{~m}$ from the floor surface and in the centre line of the saloon. The testing areas include the gangway, vestibule and saloon. The interval of the adjacent measuring points is about $1 \mathrm{~m}$. During the data collection process, the interior doors of saloon were closed. Combined with the real vehicle experiment, the impact range of wheel-rail noise and aerodynamic noise in the bogie area was confirmed according to the following two principles:

1. There has an obvious and continuous decreasing trend in noise at adjacent testing points;

2. The sound pressure level at some testing points is $3 \mathrm{~dB}(\mathrm{~A})$ or much lower than that at the centre of the sound source, which means that the sound energy is reduced by at least $50 \%$.
Based on the curve in Fig. 1, the influence area of the noise from bogie is within the range of $7 \mathrm{~m}$ from the vehicle end.

\subsection{Finite element modelling of vehicle}

According to the affecting area of the noise from a bogie, a 7-m-long vehicle body was built in accordance with the real bogie area of high-speed trains, and both of the vehicle ends were installed with outside end walls for easy sealing.

As for high-speed trains, the vehicle is a structure mainly made up of thin plate. Considering the complex vehicle structure and characteristics of the vehicle profile, the vehicle was simplified into shell elements structure in order to improve the modelling efficiency and reduce calculation time. At the same time, some components were neglected, such as transition fillet, which has little impact for the structural performance. ABAQUS software was adopted in finite element modelling. Based on the detailed analysis of vehicle grid, the global grid cell was meshed with a length of $60 \mathrm{~mm}$. Of them, there are 120,781 quadrilateral units and 4206 triangular units, accounting for $3.48 \%$ of all cells. The finite element model of the vehicle is shown in Fig. 2.

Since the vehicle structure was simplified in the finite element modelling process, it was necessary to verify the accuracy of the finite element model through modal test.

In the modal test, the test vehicle body was first supported by elastic rubber in order to test modal parameters in the approximately free state, as shown in Fig. 3. Then, the excitation signal was generated by the electromagnetic exciter, and the data were collected simultaneously through dynamic data acquisition system. Finally, the modal analysis software was used to analyse the test vehicle body mode frequency, and the first several modal frequencies and mode shapes are extracted as needed.

The calculated and experimental mode results are given in Table 1 and shown in Fig. 4.

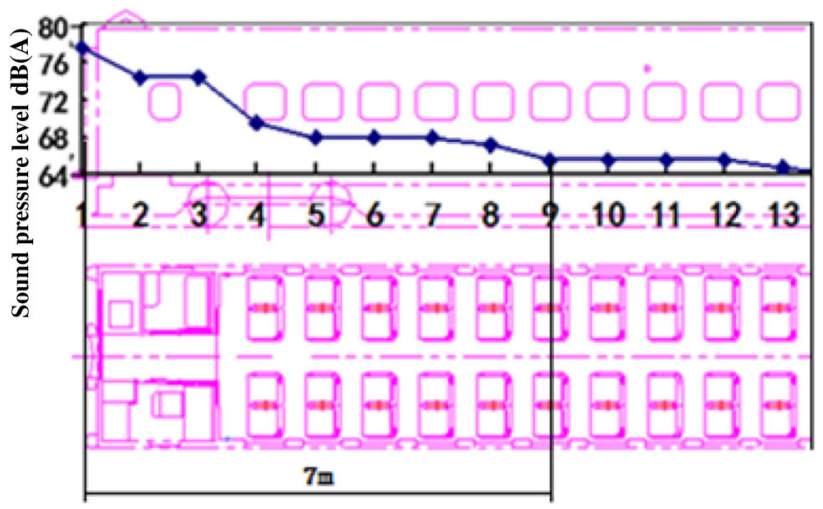

Fig. 1 Longitudinal distribution of interior noise 


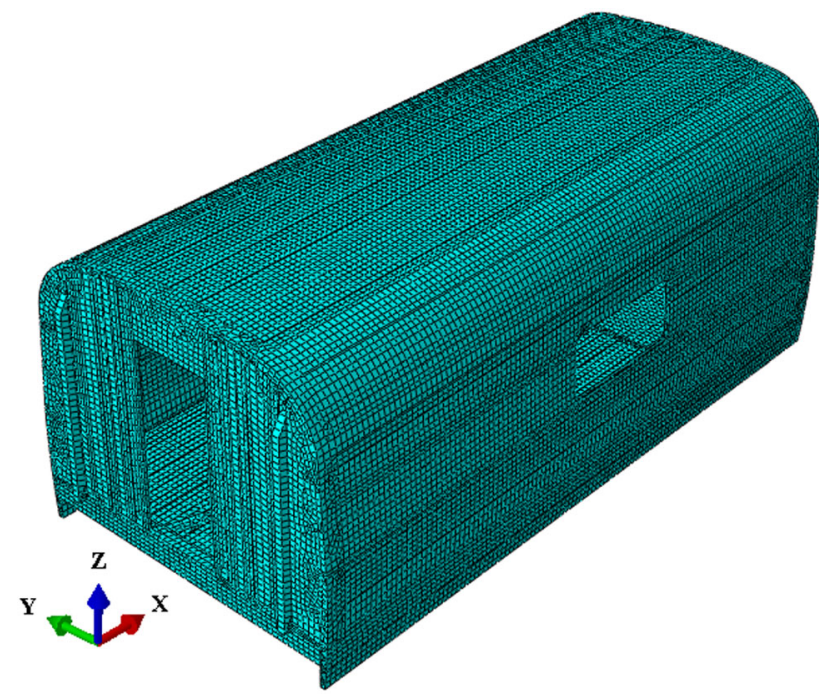

Fig. 2 Finite element model of vehicle

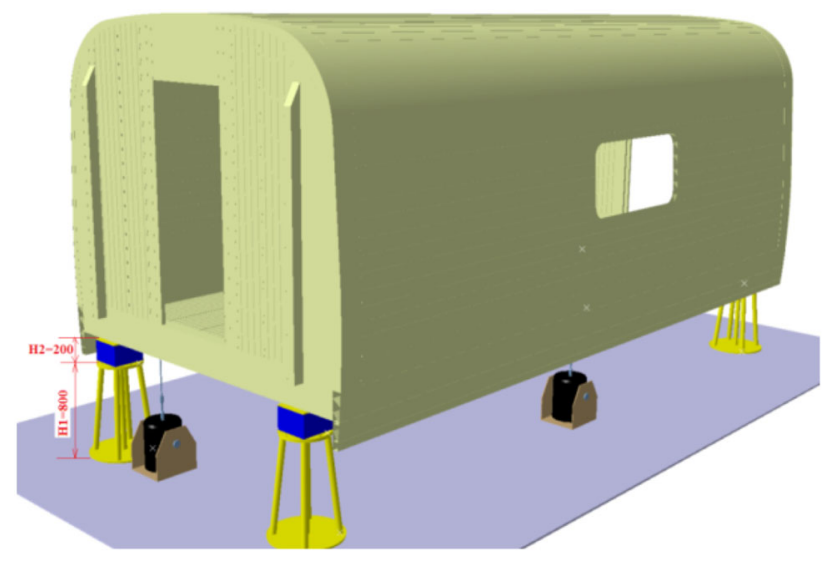

Fig. 3 Elastic rubber support of real vehicle platform

Table 1 and Fig. 4 show that the natural frequency errors between the calculated modal and the experimental platform are within $6 \%$, and the modal vibration shapes are consistent. This indicates that the established finite element model of the vehicle can accurately reflect the vibration characteristics of the real vehicle structure. It can be used for subsequent analysis of random vibration and acoustic radiation.

\section{Acquisition of random vibration standard spectrum in bogie area}

Reference [9] shows that the main vibration and noise sources of high-speed trains come from the bogie area. The vibration and noise of the bogie area mainly come from wheel-rail excitation and aerodynamic excitation. From
Table 1 Comparison of modal frequency between test and simulation

\begin{tabular}{llcr}
\hline Order & $\begin{array}{l}\text { Test mode frequency } \\
(\mathrm{Hz})\end{array}$ & $\begin{array}{l}\text { Simulation mode } \\
\text { frequency }(\mathrm{Hz})\end{array}$ & $\begin{array}{l}\text { Error } \\
(\%)\end{array}$ \\
\hline 1 & 33.04 & 32.72 & -0.98 \\
2 & 50.09 & 50.62 & 1.05 \\
3 & 52.52 & 55.57 & 5.49 \\
4 & 56.85 & 56.87 & 0.03 \\
5 & 65.66 & 66.65 & 1.49 \\
6 & 71.61 & 70.78 & -1.17 \\
7 & 77.6 & 74.10 & -4.72 \\
8 & 84.9 & 88.02 & 3.54 \\
9 & 96.67 & 95.04 & -1.72 \\
10 & 104.92 & 107.47 & 2.37 \\
\hline
\end{tabular}

the perspective of energy contribution, the wheel-rail excitation and aerodynamic excitation generally keep increasing with the speed, but the energy contribution level of wheel-rail excitation and aerodynamic excitation changes at the speed of $250 \mathrm{~km} / \mathrm{h}$, and the wheel-rail excitation is dominant below $250 \mathrm{~km} / \mathrm{h}$. By contrast, aerodynamic excitation is dominant above $250 \mathrm{~km} / \mathrm{h}$ [12]. Therefore, the vibration and noise characteristics of the bogie area are the combination result of several factors including wheel set, track, frame structure, equipment, airflow and external environment.

Based on the principle of signal acquisition, we collected and selected the vibration acceleration data of 14 representative points in the bogie area with the actual operating speed of $350 \mathrm{~km} / \mathrm{h}$, and the PSD of these data was standardized as an input excitation condition. Compared with the ordinary vibration excitation spectrum, the random vibration excitation spectrum can cover and reflect the vibration characteristics of different positions in the bogie area under high-speed conditions.

Before the data processing, the selected vibration data were pre-processed and tested to analyse the basic characteristics, including stationarity, ergodicity, periodicity and normality. Then, the power density function was calculated with the Welch method.

The extreme envelope method [13] adopts the maximum spectrum to describe the random vibration environment. The maximum spectrum is composed of the maximum value of the spectral density at each frequency. In the actual calculation, frequency is not usually calculated one by one, but it is divided with a certain bandwidth. The standard spectrum processed with the extreme envelope method is shown in Fig. 5. 

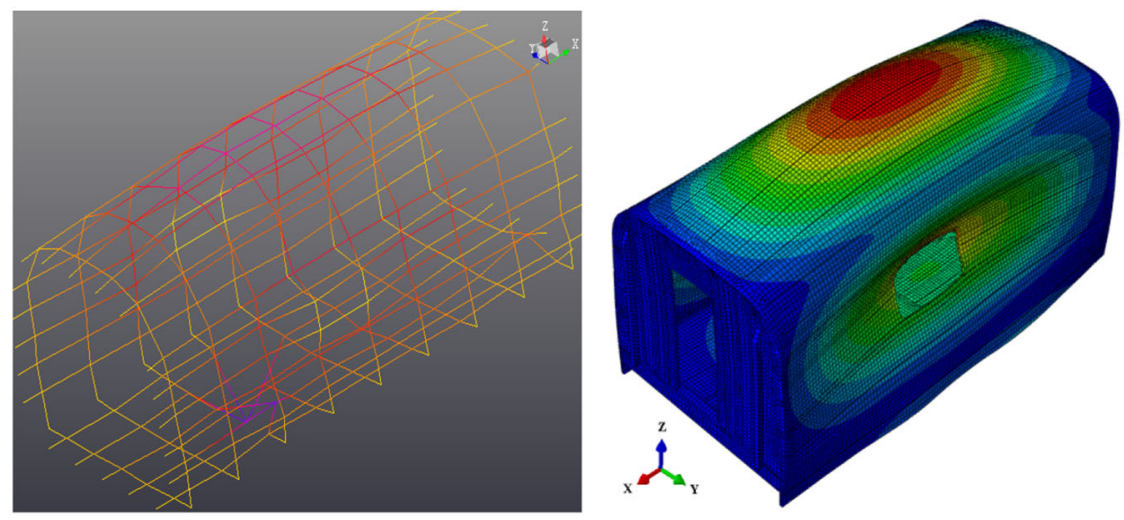

First order of test: $33.04 \mathrm{~Hz}$

First order of simulation: $32.72 \mathrm{~Hz}$
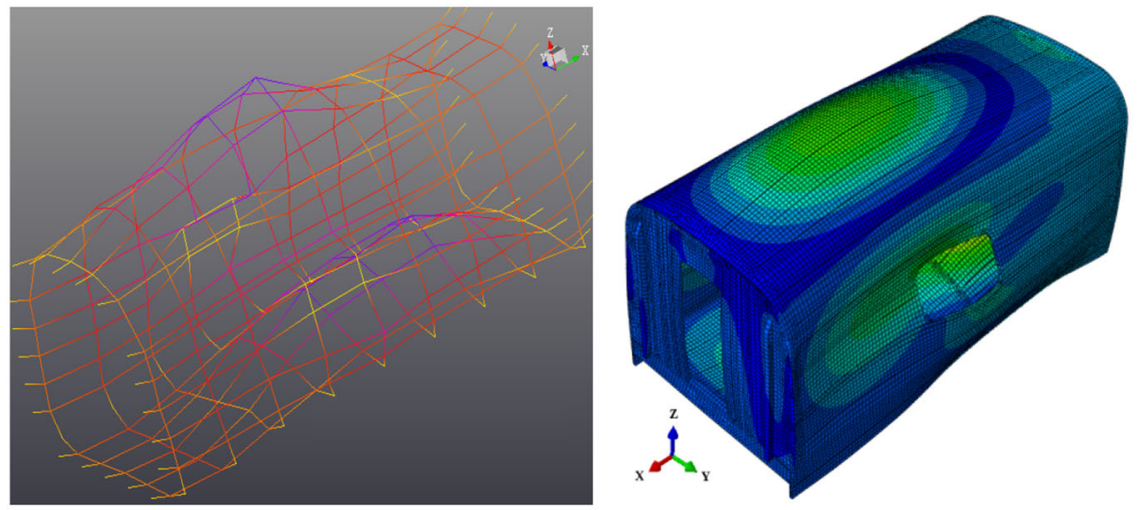

Second order of test: $50.09 \mathrm{~Hz}$

Second order of simulation: $50.62 \mathrm{~Hz}$
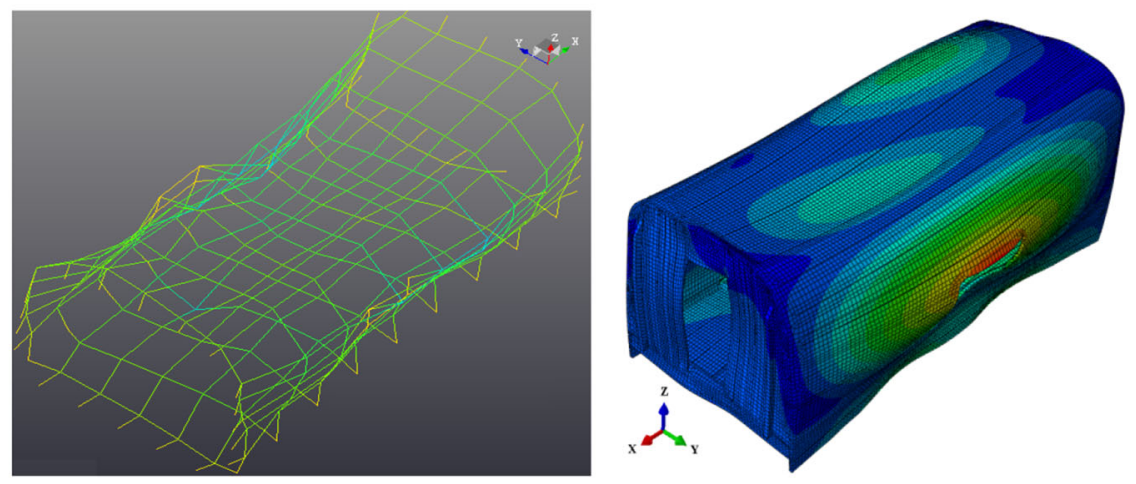

Third order of test: $52.52 \mathrm{~Hz}$

Third order of simulation: $55.57 \mathrm{~Hz}$
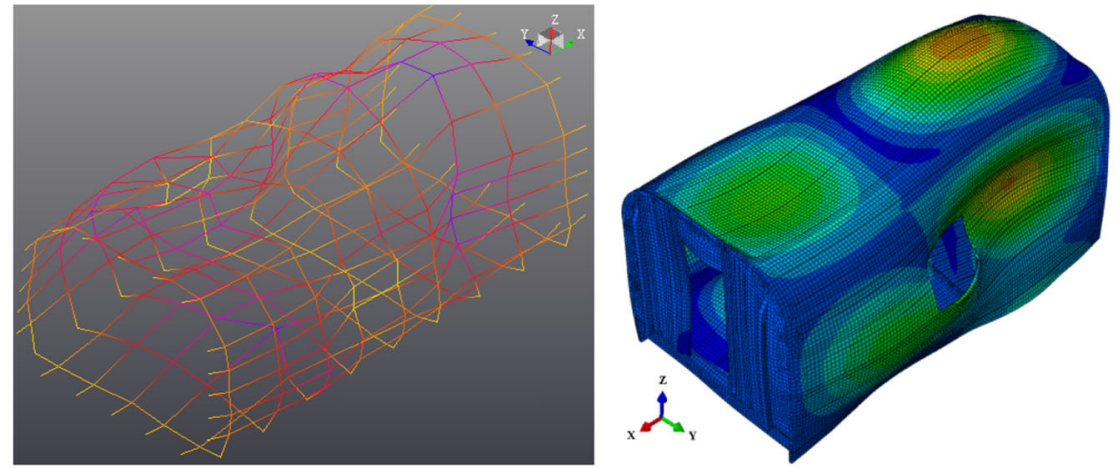

Fourth order of test: $56.85 \mathrm{~Hz}$

Fourth order of simulation: $56.87 \mathrm{~Hz}$

Fig. 4 Comparison of modal shape between test and simulation 


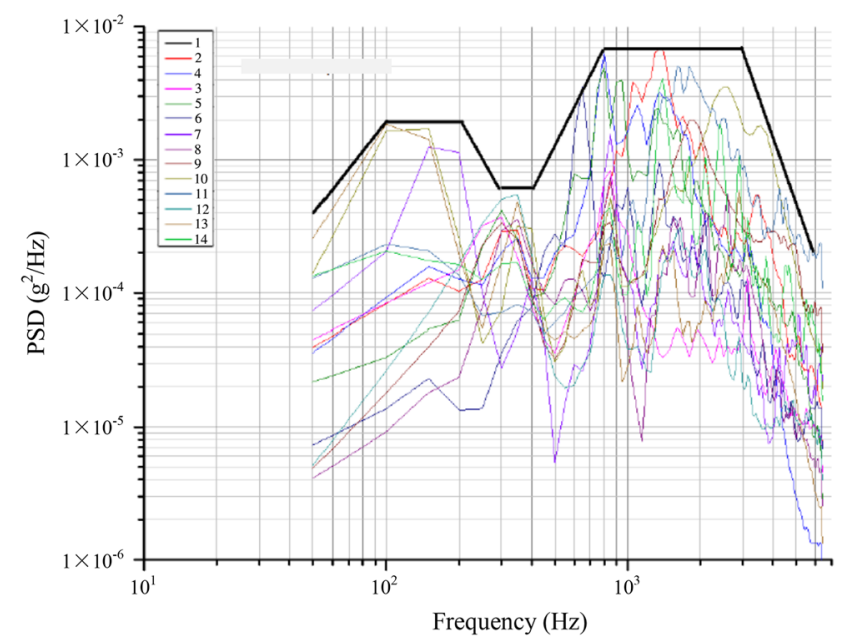

Fig. 5 Standard spectrum of PSD curves at measuring points based on extreme envelope method

\section{Random vibration prediction and analysis of real vehicle structure platform}

\subsection{Random vibration test by real vehicle platform}

In order to further verify the accuracy of the random vibration and noise prediction model of the real vehicle platform, the random vibration and noise test was carried out. The electric vibration table is ES-10-240 produced by the Dongling Corporation, and a special jig was designed to connect the vehicle body and vibration platform, and four elastic supports were installed on both sides of the beam at the end of the vehicle. The control conditions of random vibration test are given in Table 2. Before the formal test, the pretest for the key frequency band was performed to understand the vibration characteristics of the system and observe the control curve. The installation of the test body
Table 2 Random vibration test conditions

\begin{tabular}{lllll}
\hline Frequency $(\mathrm{Hz})$ & PSD $(\mathrm{Hz})$ & RMS $(\mathrm{g})$ & Load time $(\mathrm{s})$ & Direction \\
\hline 20 & $1 \mathrm{e}-5$ & 0.136 & 240 & Vertical \\
100 & $5 \mathrm{e}-5$ & 0.136 & 240 & Vertical \\
300 & $5 \mathrm{e}-5$ & 0.136 & 240 & Vertical \\
500 & $1 \mathrm{e}-5$ & 0.136 & 240 & Vertical \\
\hline
\end{tabular}

on the test platform is shown in Fig. 6. In this test, 24 measuring points were separately arranged on the roof, side plate and the floor. An acoustic sensor was placed in the middle of the vehicle, which is $1.2 \mathrm{~m}$ above the floor. Since the background noise generated by the cooling fan was large, an acoustic sensor was arranged near the fan. The arrangement of the measuring points is shown in Fig. 7.

\subsection{Random vibration simulation and analysis}

According to the excitation position in the vehicle platform test, the random excitation was loaded at the corresponding position of the simulation model. The calculated frequency range was $20-200 \mathrm{~Hz}$, and the step size was $1 \mathrm{~Hz}$. The obtained random vibration control curve is shown in Fig. 8 . Comparison of the PSD curves of partial vibration measurement points for simulation and test is shown in Fig. 9.

Figure 8 shows that the random vibration control curves are all within the $3 \mathrm{~dB}$ tolerance, which indicates that the vibration test precision is desirable.

Figure 9 shows that the amplitude of PSD curves for simulation and experimental test is basically consistent at the primary peak, but there are certain differences in the amplitude and the secondary peak frequency. This is due to the simplification of the model and the setting of the modal damping parameters.
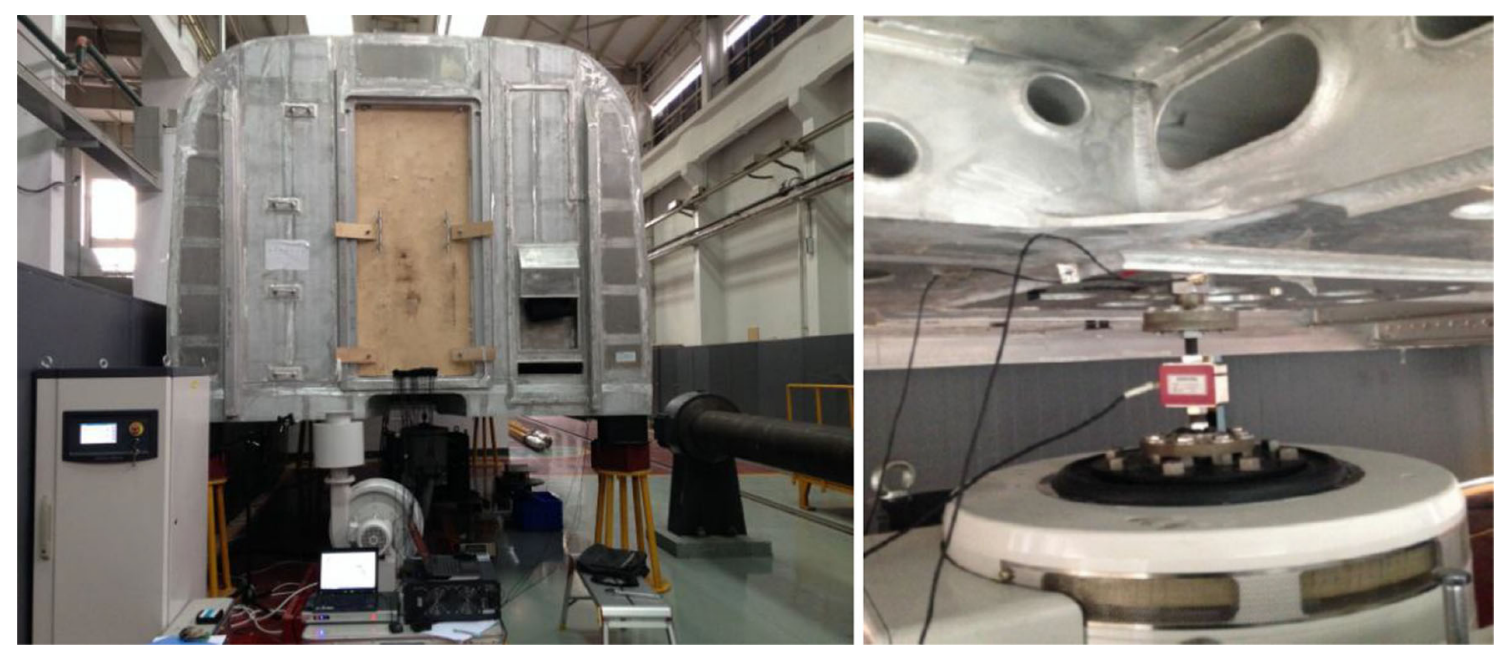

Fig. 6 Installation of exciter and the scene for test platform 

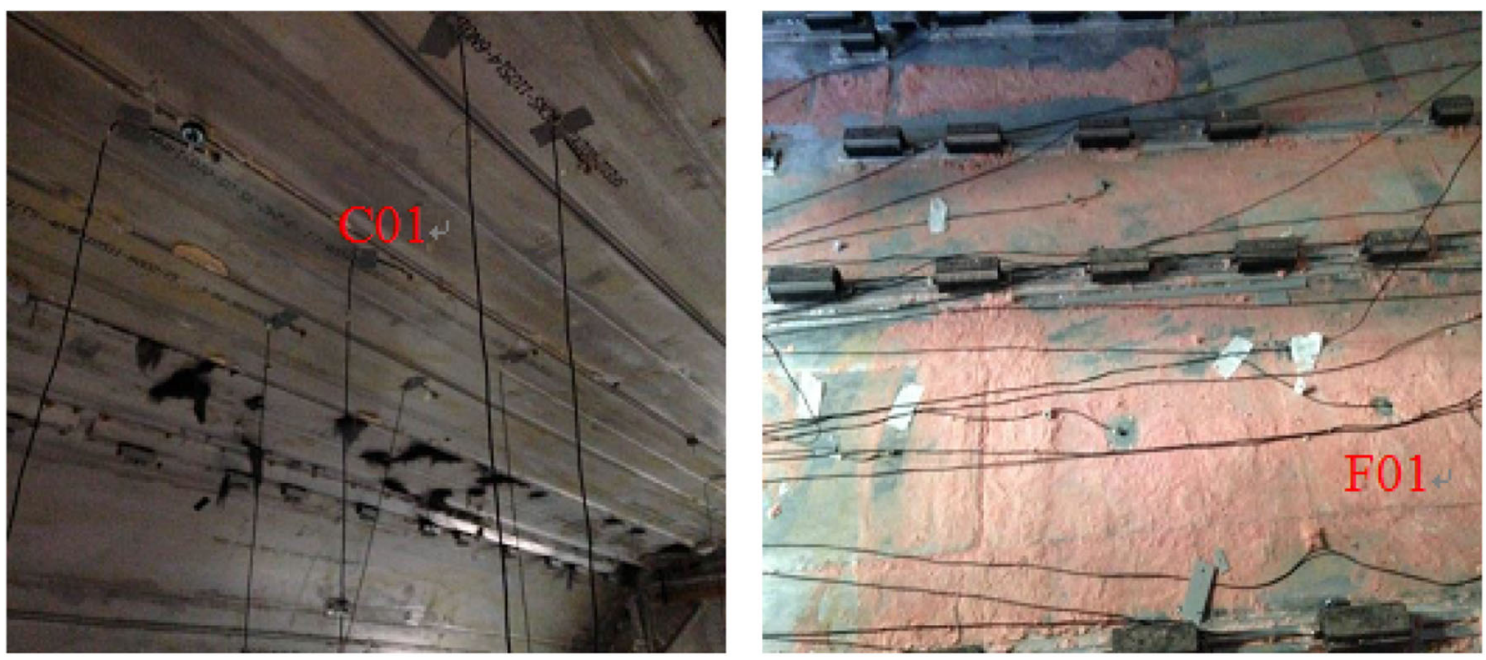

(a) Arrangement of vibration measuring points
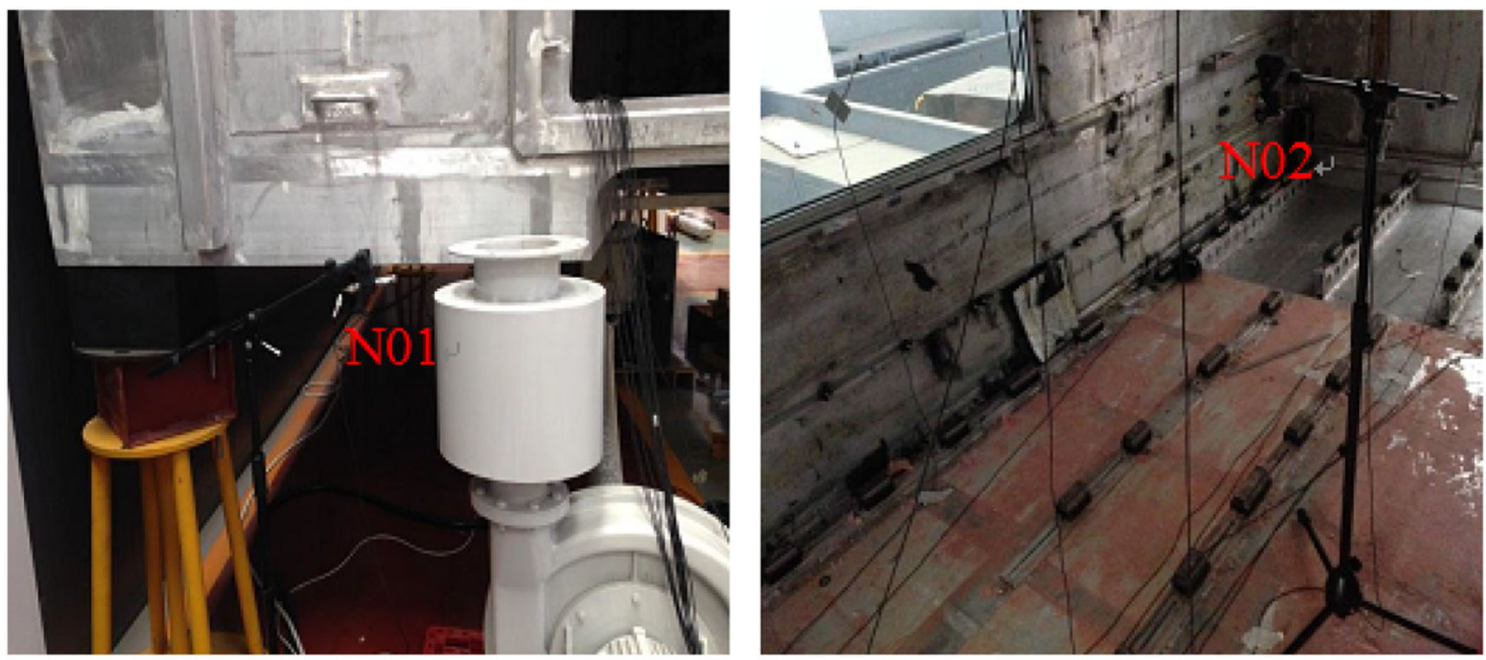

(b) Arrangement of noise measuring points

Fig. 7 Measuring points of vibration and noise test (C01: vehicle top, F01: vehicle floor, N01: outside of the vehicle, N02: inside of the vehicle)

From the test and simulation results, in the frequency range of $60-110 \mathrm{~Hz}$, the vehicle has relatively high vibration energy, so the reasonable control measures in this frequency band can reduce vibration and noise.

\section{Noise prediction and analysis of interior}

\subsection{Finite element modelling of vehicle acoustic boundary}

The vehicle acoustic cavity model is a model of internal space, which is surrounded by the ceiling, side plates, floor, front and rear sections, etc. Based on the present structural model, the inner panels were used as the outer surface of the acoustic cavity model. In order to ensure the accuracy of the structure, at least 6 acoustic units must be included in each acoustic wavelength range [14]. Since the calculated upper frequency limit was set as $500 \mathrm{~Hz}$, the meshed unit length was set to be $100 \mathrm{~mm}$ through the pre-calculation of the body cavity model. The acoustic model after meshing is shown in Fig. 10, and it has a total of 9486 nodes and 9588 units, of which QUAD4 unit is the main unit.

\subsection{Load excitation}

In the random vibration test, the acceleration PSD was directly loaded at the excitation position through the vibration table, but in the process of the acoustic vibration analysis with LMS Virtual Lab, it cannot be directly loaded. In order to achieve the same loading effect as in the test, the large mass method was adopted, which was to set a 


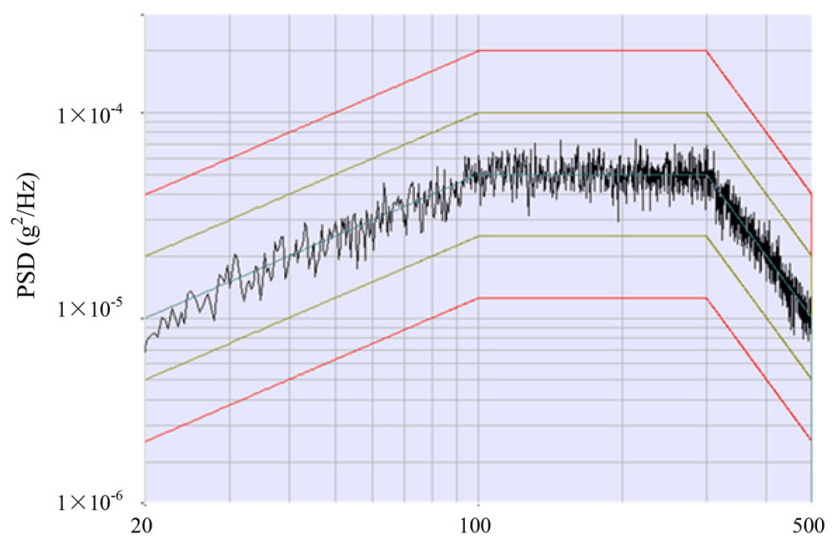

Fig. 8 Random vibration control curve

mass point with big weight at the position of the excitation source (about $10^{4}$ to $10^{8}$ times of the body model; $10^{6}$ was used in this paper). The loading position for the simulation and test excitation was located at the centre pin of main frame beam of the vehicle body. The connection position is shown in Fig. 11.

\subsection{Prediction and analysis of interior noise}

The indirect boundary element method was adopted to predict the acoustic response of the vehicle platform. Unlike the direct boundary element method, the indirect boundary element method only needs to determine the velocity difference $\sigma$ and sound pressure difference $\mu$ on both sides of the boundary element model. The sound pressure of the observation point is expressed as

$p(r)=\int_{s_{x}}\left[G\left(r, r_{Q}\right) \sigma\left(r_{Q}\right)-\frac{\partial G\left(r, r_{Q}\right)}{\partial \boldsymbol{n}_{Q}} \mu\left(r_{Q}\right)\right] \mathrm{d} S_{Q}$,

where $p(r)$ is sound pressure level of the observation point; subscript $Q$ represents the source point on the surface of the boundary element; $\boldsymbol{n}$ is the unit normal vector at the source point; $G\left(r, r_{Q}\right)=\mathrm{e}^{-\mathrm{i} k\left|r-r_{Q}\right|} / 4 \pi\left|r-r_{Q}\right|$ is Green's function; $k=w / c$ is wave number; $w$ is circle frequency of vibration; $c$ is fluent sound velocity; and $\sigma\left(r_{Q}\right)$ and $\mu\left(r_{Q}\right)$, respectively, represent the normal velocity difference and the pressure difference on both sides of the boundary element model surface, which are expressed as

$\sigma\left(r_{Q}\right)=\frac{\partial p\left(r_{Q_{1}}\right)}{\partial n}-\frac{\partial p\left(r_{Q_{2}}\right)}{\partial n}=-\mathrm{i} \rho \omega\left(v_{n}\left(r_{Q_{1}}\right)-v_{n}\left(r_{Q_{2}}\right)\right)$,

$\mu\left(r_{Q}\right)=p\left(r_{Q_{1}}\right)-p\left(r_{Q_{2}}\right)$.

In Eq. (2), $p\left(r_{Q 1}\right)$ and $p\left(r_{Q 2}\right)$ are the pressures on both sides of the structural surface; $v_{n}\left(r_{Q 1}\right)$ and $v_{n}\left(r_{Q 2}\right)$ are the normal vibrational speeds on both sides of the structural

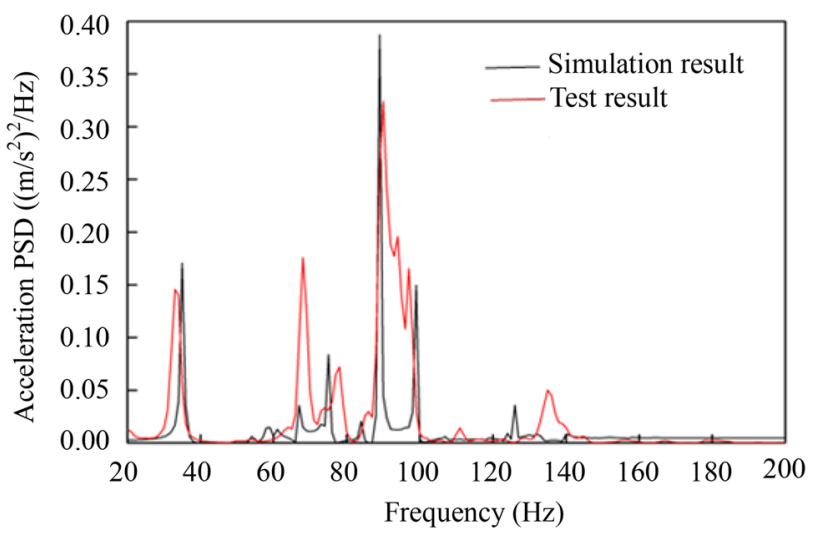

(a) At C01

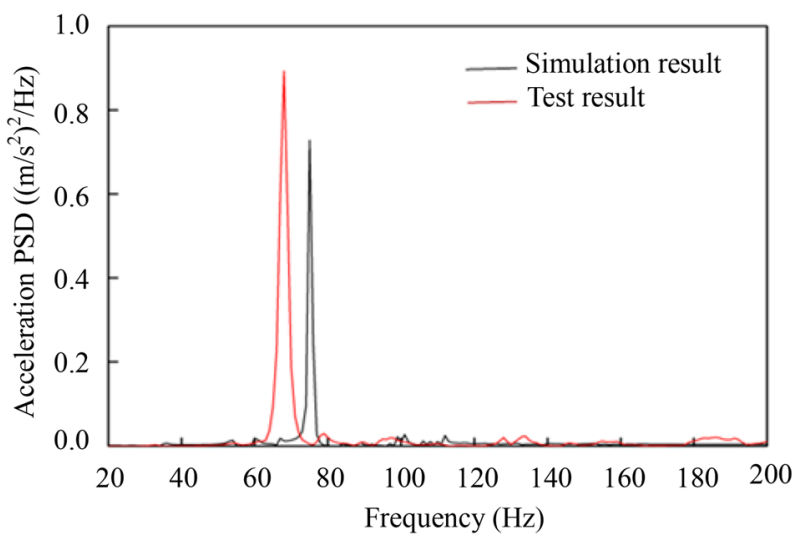

(b) At F01

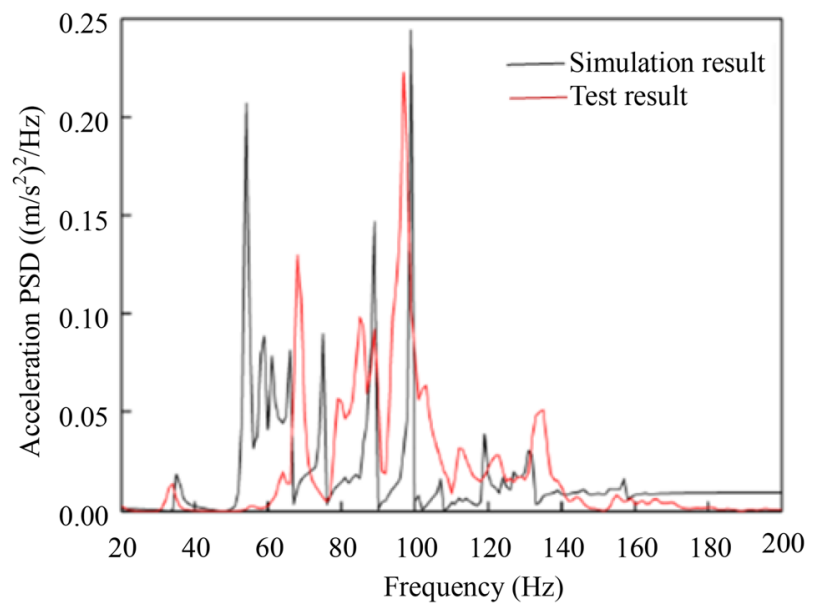

(c) At F02

Fig. 9 Comparison of vibration acceleration PSD curves at measuring points

surface. It is assumed that the boundary element model surface satisfies the Neumann boundary condition, the measurement point $P$ is located on the model boundary, and the position vector is $\boldsymbol{r}_{P}$. The relationship between the 

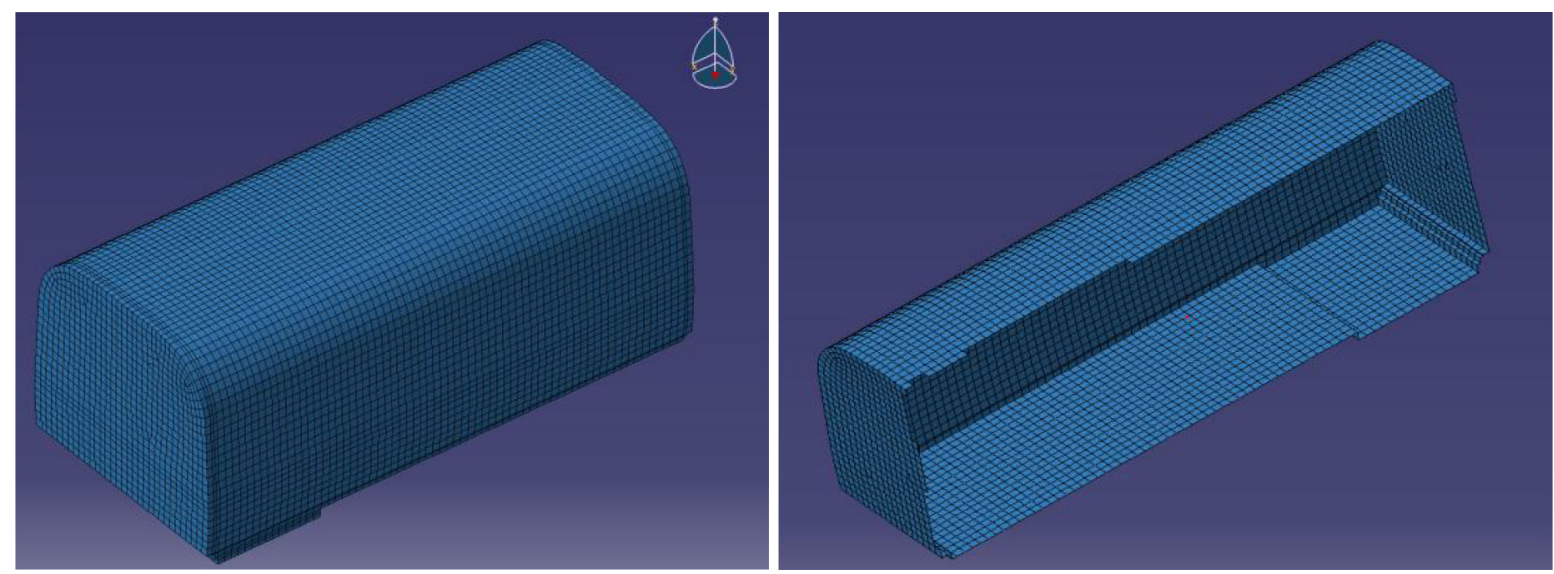

Fig. 10 Boundary of vehicle acoustic model after meshing

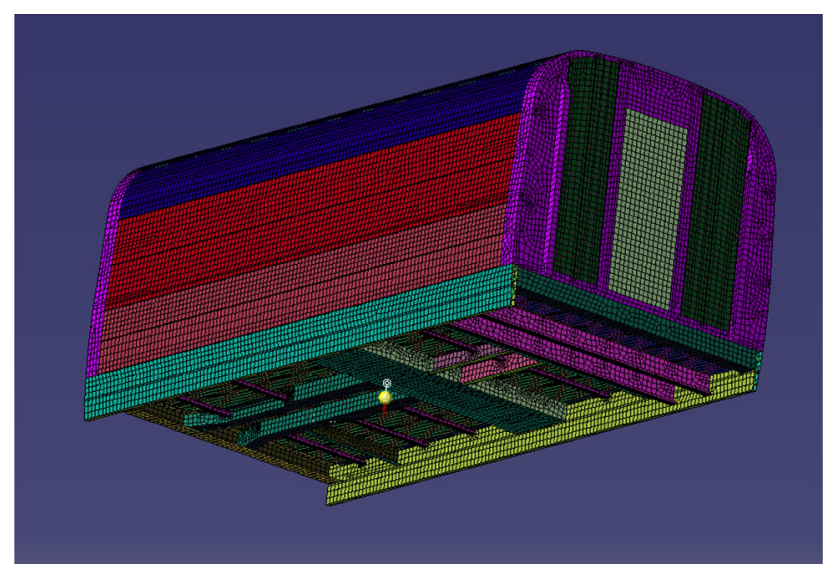

Fig. 11 Finite element model of vehicle body with a large mass point

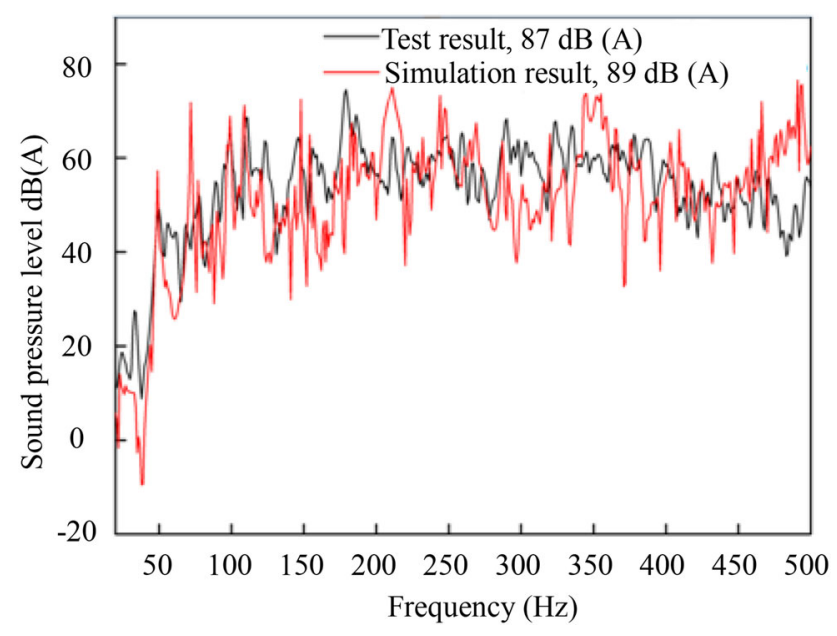

Fig. 12 A-weighted sound pressure level at N02 of test and simulation boundary condition of the model and the unknown main variables is

$$
\begin{aligned}
\frac{\partial p\left(\boldsymbol{r}_{P}\right)}{\partial \boldsymbol{n}_{P}} & =\int_{s_{Q}}\left[\frac{G\left(\boldsymbol{r}_{P}, r_{Q}\right)}{\partial \boldsymbol{n}_{P}} \sigma\left(r_{Q}\right)-\frac{\partial G\left(\boldsymbol{r}_{P}, r_{Q}\right)}{\partial \boldsymbol{n}_{P} \cdot \partial \boldsymbol{n}_{Q}} \mu\left(r_{Q}\right)\right] \mathrm{d} S_{Q} \\
& =-\mathrm{i} \rho \omega v_{n}\left(\boldsymbol{r}_{P}\right)
\end{aligned}
$$

where $v_{n}\left(\boldsymbol{r}_{P}\right)$ is the normal vibration velocity at point $P$ on the surface of the boundary element acoustic model and $\rho$ is the fluid density.

Combined with the theory of random vibration and acoustic radiation, the spectral density function of the structural model is $S_{i, j}(\omega), i, j=1,2, \ldots, m$, where $m$ is the number of random excitations. When $i=j, S_{i, j}(\omega)$ is autopower spectral density function; when $i \neq j, S_{i, j}(\omega)$ is the cross-power spectral density function. When the acoustic frequency response function $P\left(\boldsymbol{r}_{P}\right)$ and the PSD function $S_{i, j}(\omega)$ are known, the PSD function of each domain point can be obtained, which can be expressed as

$S_{p_{1} p_{2}}(\omega)=\sum_{i=1}^{m} \sum_{j=1}^{m} p\left(r_{p_{1_{i}}}\right) p^{*}\left(r_{p_{2_{j}}}\right) S_{i, j}(\omega)$,

where $P_{1}$ and $P_{2}$ refer to any two observation points; $S_{p_{1} p_{2}}(\omega)$ is the mutual spectral density function of $P_{1}$ and $P_{2}$; if each excitation is uncorrelated, $S_{i, j}(\omega)=0(i \neq j)$. If there is only one observation point $P$, the self-spectral density function $S_{p p}(\omega)$ of the sound pressure response at point $P$ can be obtained.

After obtaining the spectral density function of sound pressure response according to Eq. (4), the sound pressure level of each point in design field can be obtained:

$L=10 \log _{10} \frac{\left|S_{p_{1} p_{2}}(\omega)\right|}{P_{\mathrm{ref}}^{2}}$, 
where $P_{\text {ref }}=2 \times 10^{-5}$ is the reference sound pressure.

The calculated frequency range was from 20 to $500 \mathrm{~Hz}$, and the calculation step was $1 \mathrm{~Hz}$. The comparison of the A-weighted sound pressure level between the simulation and test at the standard point (N02) is shown in Fig. 12.

Figure 12 shows that the A-weighted sound pressure level curves of the simulation prediction and test nearly coincide, but at some frequencies, there is difference in the amplitude of sound pressure level. This is mainly caused by the following factors. First of all, the background noise and incomplete sealing of the front and rear doors may still cause fluctuation in noise test. Secondly, because the body structure model was simplified, it was difficult to completely approximate to an actual vehicle structure. Though the random vibration control curve was within the allowable range, it still had a certain deviation from the simulated excitation curve. Finally, there is a certain error in setting the body modal damping.

In general, however, the difference between the simulated prediction and the experimental values is $2 \mathrm{~dB}(\mathrm{~A})$, which is within error tolerance. Figure 12 shows that the peak frequencies of the A-weighted sound pressure level curve mainly appear in the ranges of $60-110,175-250$ and $340-360 \mathrm{~Hz}$. Of them, $60-110 \mathrm{~Hz}$ is mainly caused by structural modal resonance. The peaks of sound pressure level in the frequency ranges of $175-250$ and $340-360 \mathrm{~Hz}$ are closely related to the acoustic cavity vibration.

\section{Conclusions}

1. Through the PSD standardization on the line test data of the actual vehicle, the excitation condition can be obtained for the vibration and noise characteristics analysis of the vehicle structure.

2. Through modal simulation and test, and random vibration prediction and experimental analysis of the vehicle, it can be known that the vibration energy of the vehicle is mainly concentrated in the frequency range of $60-110 \mathrm{~Hz}$. If the vibration energy in this frequency band can be reasonably controlled, vibration and noise can be reduced.

3. By comparing the interior noise prediction and experimental results of the real vehicle platform, the error between the simulated prediction and experimental values is within the allowable range, and the main peak frequencies of the noise are determined; the resonance of the structural mode and the acoustic cavity mode is the main cause of the peak of sound pressure level.
Acknowledgements The authors acknowledge the support for this work from the Ministry of Science and Technology of China (2016YFB1200500).

Open Access This article is distributed under the terms of the Creative Commons Attribution 4.0 International License (http:// creativecommons.org/licenses/by/4.0/), which permits unrestricted use, distribution, and reproduction in any medium, provided you give appropriate credit to the original author(s) and the source, provide a link to the Creative Commons license, and indicate if changes were made.

\section{References}

1. Liu Z, Chen XZ (1997) Statistical boundary element method for acoustic radiation of random vibration structures. J Acoust 22(6):495-500

2. Allen MJ, Vlahopoulos N (2000) Integration of finite element and boundary element methods for calculating the radiated sound from a randomly excited structure. Comput Struct 77(2):155-169

3. Allen MJ, Vlahopoulos N (2001) Noise generated from a flexible and elastically supported structure subject to turbulent boundary layer flow excitation. Finite Elem Anal Des 37(9):687-712

4. Li XF, Zhang J, Zhao WZ et al (2007) Research on acoustic numerical simulation for structure-acoustic coupled random vibration. J Syst Simul 19(8):1683-1685

5. Bao-shan LIU, Guo-zhong ZHAO, Rui-yong LI et al (2011) Pem based acoustic radiation analysis from random excited structures. Chin J Solid Mech 32(6):581-587

6. Bao-shan LIU, Guo-zhong ZHAO (2011) Sensitivity analysis and design optimization of acoustic radiation from random excited structures. J Vib Eng 24(3):309-314

7. Zhao GZ, Chen G, Kang Z (2012) An iterative algorithm for analysis of coupled structural-acoustic systems subject to random excitations. Acta Mech Sin 28(2):458-467

8. Mehran S, Davood Y (2015) Vibro-acoustic analysis of a coach platform under random excitation. Thin Walled Struct 95:287-296

9. Mellet C, Letourneaux F, Poisson F et al (2006) High speed train noise emission: latest investigation of the aerodynamic/rolling noise contribution. J Sound Vib 293(3/4/5):525-546

10. Su-ming XIE, Tao LI, Xiao-feng LI et al (2009) Prediction on the vibration and noise of high-speed train structure and research on the noise reduction technology. China Railw Sci 30(6):77-83

11. Min XD, Zuo YY, Zhuang T (2014) Prediction and analysis of the interior noise of high-speed train. Sci Technol Eng 14(5):305-308

12. Working Group Railway Noise of the European Commission (2003) Position paper on the European strategies and priorities for railway noise abatement

13. Jun-yong SHAN, Wan-zheng BAO (1999) Recommendation for a set of vibration test conditions for land trailer-borne equipment. Fire Control Radar Technol 28(1):65-72

14. Li ZG (2005) The details of SYSNOISE Rev 5.6. National Defense Industry Press, Beijing 